\title{
Glyco-functionalised quantum dots and their progress in cancer diagnosis and treatment
}

\author{
Jayshree Ashree', Qi Wang', Yimin Chao (ه) ${ }^{1}$ \\ 1 School of Chemistry, University of East Anglia, Norwich, NR4 7TJ, UK \\ 2 Norwich Medical School, University of East Anglia, Norwich, NR4 7TJ, UK
}

(C) The Author(s) 2019. This article is published with open access at link.springer.com and journal.hep.com.cn 2019

\begin{abstract}
Despite all major breakthroughs in recent years of research, we are still unsuccessful to effectively diagnose and treat cancer that has express and metastasizes. Thus, the development of a novel approach for cancer detection and treatment is crucial. Recent progress in Glyconanotechnology has allowed the use of glycans and lectins as bio-functional molecules for many biological and biomedical applications. With the known advantages of quantum dots (QDs) and versatility of carbohydrates and lectins, Glyco-functionalised QD is a new prospect in constructing biomedical imaging platform for cancer behaviour study as well as treatment. In this review, we aim to describe the current utilisation of Glyco-functionalised QDs as well as their future prospective to interpret and confront cancer.
\end{abstract}

Keywords carbohydrate, leptin, glyco-functionalised $\mathrm{QD}$, bioimaging, cancer diagnosis and treatment

\section{Introduction}

Cancer is one of the leading causes of death and a major threat to public health in the 21 st century. Cancer is a complex type of disease affecting a verity of tissues. It is mainly characterised by uncontrolled growth of abnormal cells, with the ability to attack surrounding tissues and possibly metastasize. Despite of early stage diagnosis and therapeutics help to manage the disease, it remains to be never ending battle to prevent and treat cancer. Nanotechnology is a promising approach in diagnosis and treatment of cancer through the development of novel diagnostic imaging methods and targeted therapies. Quantum dots (QDs) are being intensively studied as a novel probe for biomedical imaging both in vitro and in vivo due to their

Received March 25, 2019; accepted May 11, 2019

E-mail: y.chao@uea.ac.uk unique optical and electronic properties. Extensive researches on the physicochemical properties of QDs, such as size, morphology, composition, and surface features suggest that QDs have great potential in cancer detection and treatment [1-6]. When conjugated with antibodies, peptides or other small biological molecules, QD-based probes can be used to target cancer cells with high specificity and sensitivity.

During the last decade, there has been a great deal of interest in the incorporation of nanotechnology with carbohydrates. Carbohydrates are prominently exposed on the surface of living cells and are critically involved in cellcell interactions and regulate important biological functions such as cell growth and cell differentiation [7]. Cancer cells differ from normal cells in many aspects, which are often exploited as a strategy in cancer chemotherapy. Cancer cell undergoes a high rate of glycolysis to adapt the low oxygen environment, leading to increased glucose demand called Warburg effect. High glucose demand leads to overexpression of insulin independent glucose transporter-1 (GLUT-1). Overexpressed GLUT-1 can be a potential target for chemotherapy. Glycoconjugates and carbohydrate derivatives can be effectively used for chemotherapy and also, conjugation of sugars to a known anti-cancer agent can increase the selectivity and hence reduce the toxicity [8]. The advances in nanotechnology have allowed the creation of different bioactive glyco-functionalised nanostructures for various biomedical applications such as drug delivery, gene therapy, pathogen detection and lectin-based biosensors [9-15].

Lectins were first introduced at the end of 19th century and are described as carbohydrate-binding proteins [16]. Lectins play important role in many biological process and living organism $[17,18]$. They are universally spread in nature and are also major component of the outer surface of mammalian cells. They have been widely used in researches involving carbohydrates recognition and detection on cell surfaces $[19,20]$. Several studies have 
conjugated carbohydrates and lectins to different nanostructures, such as polymers, liposomes, dendrimers, carbon nanotubes, metal nanoparticles and quantum dots $[9,14]$. The resulting glyco-functionalised nanoparticles have been used for cell imaging, cell separation, protein detection and enzyme immobilization [15]. Glyconanoparticles presented a highly multivalent method of interaction with cell surface structure like glycoprotein and glycolipid (Fig. 1). The major function of glyconanoparticles is to serve as recognition markers [21]. In particular, carbohydrate-protein interactions on normal cells and their malignant counterparts show significant differences [22], which is important strategy in cancer chemotherapy. Although carbohydrate-protein interactions are typically very weak but possess key role to biological processes that require temporary adhesion during cell adhesion, cancer metastasis, immune response and intracellular trafficking [15]. Moreover carbohydrate perform as essential intermediate in endocytosis processes, intercellular interaction and extracellular matrix, adhesion and cell growth [23,24], fertilisation [25], and also interactions between pathogen and host [26]. The unique functionalities of carbohydrates forming densely packed cluster on the surface of nanoparticles (NPs) is known as the "glycocluster effect" $[27,28]$. These glyconanoparticles behaves in a similar manner to mimic the naturally occurring glycocalyx. Therefore, the functionalisation and engineering of these glyconanoaprticles helps to further enhance their specific reorganisation properties on multivalent scaffolds in glycoscience.

\section{Characteristic of QDs for biomedical application}

QDs are semiconductors nanocrystals, are one of the first nanotechnologies to be integrated with the biological sciences. QDs are proven to be powerful probes for fluorescence imaging and are being developed for a range of additional applications including the detection of cancer, fluorescent assays for drug discovery, single protein tracking, and intracellular reporting. QDs have distinct properties that give them their unique capabilities.

QDs were first fabricated in the 80's by Louis E. Brus [29] and the unique properties of these special nanostructures attracted interest from many fields. QDs are semiconductor nano-crystals in which excitons are confined in all three spatial dimensions. The confinement can be realised by fabricating the semiconductor in very small size, typically several hundred to thousands of atoms per particle. Due to quantum confinement effects, QDs act like artificial atoms, showing controllable discrete energy levels. QDs range from $2 \mathrm{~nm}$ to $10 \mathrm{~nm}$ in diameter and shows unique optical, physical and chemical properties. They offer great advantages over traditional organic fluorescent dyes. They present several beneficial characteristics for spectroscopy and microscopy, such as high fluorescence intensity, long lifetime, and good resistance to photobleaching. The brightness of QD based multifunctional probes affords high sensitivity for simultaneous cancer molecular imaging and targeted therapy. For spectrum application, the sensitivity of QD-based molecular imaging can be two to three orders larger than that of routine fluorescent dyes [30]. Furthermore, the fluorescence near-infrared (NIR) of NIR-QDs can be detected in deep tissues, making them suitable for in vivo imaging with high signal to background ratio. These properties are enabling a new generation of fluorescence imaging experiments in cancer research allowing investigators to unravel biological function at the molecular level. When functionalized with diagnostic and therapeutic agents, QDs can be used for cancer diagnosis, photodynamic therapy,

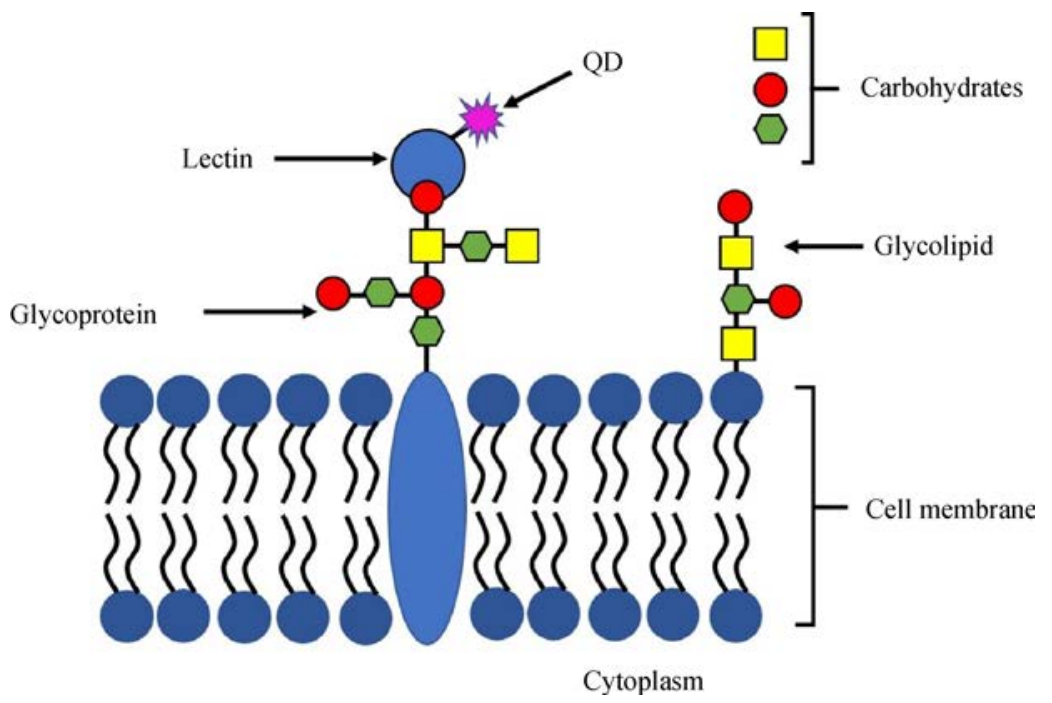

Fig. 1 Scheme of the cell membrane carbohydrate residues labelling with lectin-QDs. 
cell labelling and biosensors. Biocompatibility and biological targeting of QDs are achieved through surface modification and conjugation with antibodies, peptides, or small molecules. The glyconanoparticles shows promising results in fluorescence-based techniques for biological studies when associated with QDs to lectins or carbohydrate.

2.1 Lectin-functionalised QDs in cancer diagnosis and theranostics

In 1998, first biocompatible QDs were announced for cancer imaging in in vitro. Despite of immeasurable technological advances thereafter in cancer diagnosis, the conventional medical imaging techniques in most of cases fail to offer sensitivity and resolution altogether for early stage diagnosis, as well as providing specific disease molecular information. Signal-to-background ratio is important and needs lot of improvement in order to effectively screening, staging and treatment of cancer. To achieve that proportional improvements in sensitivity and contrast targeting agent are required. The objective of cancer imaging is to detect and image the smallest number of tumour cells, ideally before the angiogenesis [31,32]. Current detection for solid tumours has a threshold of $\sim 10^{9}$ cells [31,33]. When assisted by the administration of specific exogenous contrast agents [34-37], current imaging modalities can monitor biochemical processes as well as cross-sectional anatomy [38,39]. Though, they have certain limitations [40-42], which are either due to their intrinsic characteristics or the potentially harmful effect of the contrast agents. QD-based imaging technique could overcome these limitations, make it as one of the most promising technologies for early diagnosis of cancer $[43,44]$.

Glycans are carbohydrates that can be attached to proteins, lipids and other glycans through enzymic process known as glycosylation. Glycans have key roles in cancer biology, including cell signalling, tumorigenesis, immune modulation, angiogenesis and metastasis $[45,46]$. Aberrant changes in glycan structures have been shown to be associated with tumorigenesis, tumour progression and metastasis, thus a universal hallmark of cancer $[46,47]$. Compared to normal tissue, tumour cells have numerous changes in glycosylation, including changes to sialylation, fucosylation, the truncation of $\mathrm{O}$-glycans, and $\mathrm{N}$-glycan branching $[47,48]$. There is a huge potential to exploit glycans to improve early diagnosis, and as markers of specific therapeutic targets [45]. Lectin-QD bioconjugate could be an ideal tool to study glycan profile and glycosylation changes related to cancer. Table 1 presents a summary of Lectin functionalised QDs used for cancer diagnosis studies reported in literature.

\subsubsection{Lectin functionalised QDs in cancer diagnosis}

Andrade et al. [49] formulated CdTe QDs functionalised with MSA and conjugated with concanavalin A (Con A) or Ulex europaeus agglutinin I (UEA I) lectins to investigate conceivable variations in carbohydrates profile in normal breast tissue, fibroadenoma and invasive ductal carcinoma (malign tumour). These CdTe QDs are used to detect $\alpha$-Dglucose/mannose and L-fucose residues on cell surface. They showed to be more photostable and had higher luminescence intensity than the previously used glutaraldehyde $\mathrm{CdS} / \mathrm{Cd}(\mathrm{OH})_{2}$ QDs [54]. They observed that the tissues stained with these CdTe QD conjugates showed different fluorescent patterns, which revealed distinct labelling patterns in the three tissue types. The stroma was favourably and intensely stained by CdTe QDs-Con A, due to higher expression of $\alpha$-D-glucose/mannose residues. The ductal cells were preferentially labelled by CdTe QDsUEA I, revealing intense expression of L-fucose residues. These results indicate differences in expression and distribution of carbohydrate residues in these tissues.

He et al. [50] synthesised hydrophilic TGA functionalised CdTe QDs conjugated with Datura stramonium agglutinin (DSA) and Lens culinaris agglutinin (LCA), which have different emission wavelength of 550 and $618 \mathrm{~nm}$, respectively (Fig. 2(A)). The formed functionalised QD-lectin bioconjugates are used to investigate the GalNAc and GlcNAc/mannose profiles on hepatocellular carcinoma cells (HepG2) by fluorescence imaging (Fig. 2(B)) and flow cytometric analysis. Compared to

Table 1 Summary of lectin-functionalised QD conjugates for cancer diagnosis reported in literature

\begin{tabular}{|c|c|c|c|c|c|}
\hline Application & Carbohydrate detection & Lectin & Types of QDs & Cancer model & Ref. \\
\hline Cancer diagnosis & $\begin{array}{l}\text { Glucose/mannose } \\
\text { and L-fucose profile }\end{array}$ & Con A, UEA I & MSA-CdTe QDs ${ }^{\text {a) }}$ & $\begin{array}{c}\text { Fibroadenoma and invasive } \\
\text { ductal carcinoma cells }\end{array}$ & {$[49]$} \\
\hline Cancer detection & $\begin{array}{l}\text { GalNAc, GlcNAc } \\
\text { and mannose profiles }\end{array}$ & DSA, LCA & TGA-CdTe QDs ${ }^{\text {b) }}$ & HepG2 cells & {$[50]$} \\
\hline Cancer diagnosis & $\begin{array}{l}\text { Mannose/glucose, } \\
\text { and galactose profile }\end{array}$ & Cramoll & CdTe QDs & & {$[51]$} \\
\hline Cancer theranostics & $\begin{array}{c}\text { Mannose triflate, } \\
\text { cysteamine molecules (MTC) }\end{array}$ & SNA lectin & $\mathrm{CdSe} / \mathrm{CdS}$ & Caco-2, MCF-7 and A549 cells & {$[52]$} \\
\hline Cancer theranostics & - & PHA-L & $\mathrm{CdSe} / \mathrm{CdS}$ QDs & MCF-7 cells & {$[53]$} \\
\hline
\end{tabular}

a) MSA: Mercaptosuccinic acid; b) Thioglycolic acid. 


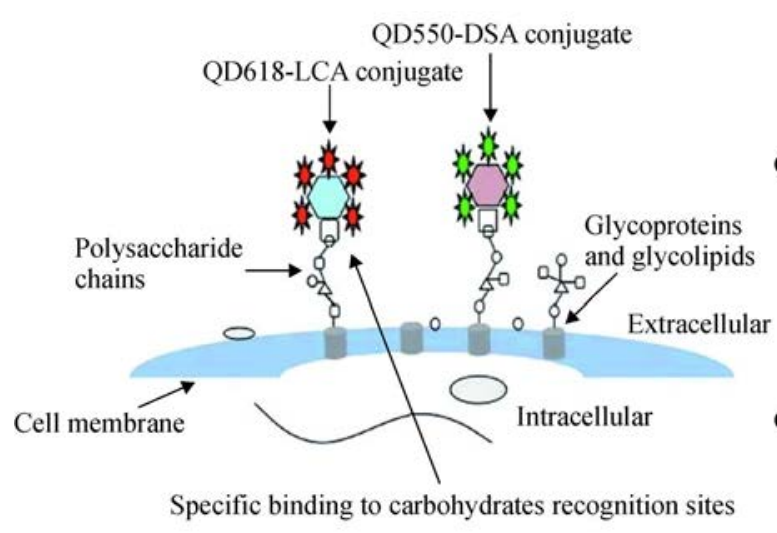

(A)

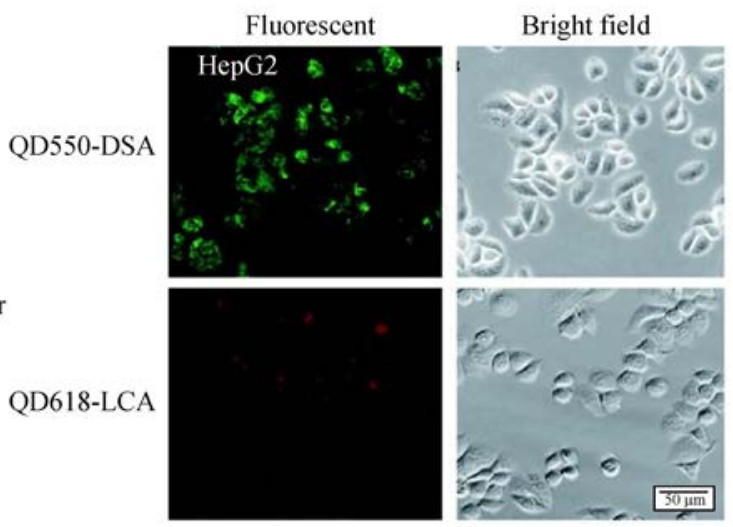

(B)

Fig. 2 (A) Schematic representation of lectin-QDs conjugates: QD550-DSA and QD618-LCA detecting cell surface carbohydrates. (B) Fluorescence microscopy images of HepG2 cells stained by functionalised QDs. (Adapted with permission from Ref. [50], published by The Royal Society of Chemistry)

normal endothelium cells (ECs) and liver cells (LO2), HepG2 cancer cells labelled with QDs-DSA and QDsLCA exhibited 3 and 2-fold greater signal, respectively. Thus, the lectin-QD bioconjugates proved to be efficient tools to monitor the carbohydrate expression and evaluating the differential expression of these carbohydrates on normal and cancer cells, which is very important for helping the early diagnosis of cancer.

A recent study from Cunha et al. [51] evaluated two different strategies to conjugate cramoll lectin to QDs. Cramoll is a mannose/glucose-binding lectin with unique immunomodulatory and antitumor activities. Both adsorption and covalent bonding strategies have been used and tested at different $\mathrm{pH}$ values. The authors showed that QDs absorbed to cramoll at $\mathrm{pH} 7.0$ had the best labelling efficiency on Candida albicans cells. Approximately $92 \%$ of cells were labelled after incubation with adsorption conjugates compared to $17 \%$ of cells were labelled by covalent conjugates. The cramoll lectin and QD conjugates remained brightly fluorescent and preserved identical biological activity as cramoll, thus it could be promising fluorescent tools for carbohydrate expression analysis in normal and cancer cells, which can provide valuable information about glycosylation changes related to cancer.

\subsubsection{Lectin functionalised QDs in theranostics for cancer}

Theranostics, is a term invented from merging diagnostics and therapeutics application of nanomaterials. QDs are excellent candidate for imaging and early cancer detection; they may also serve ideal delivery vehicles if the biocompatibility can be managed. Through surface functionalization of ligands and conjugation of "drugs" on QD one can construct an "all-in-one" multifunctional nanoplatform that features targeting, therapeutic and imaging modalities. Lectin-QDs are showing promising results in theranostics applications.
Akca et al. [52] synthesised CdSe/CdS QDs and conjugated with sambucus nigra agglutinin (SNA) lectin and MTC. Cysteamine can induce apoptosis in cells, and its capacity can be increased with radiation. Biological activities of ${ }^{125} \mathrm{I}-$, ${ }^{125} \mathrm{I}-\mathrm{MTCQDS}$, MTC-QDs-Lec- ${ }^{125} \mathrm{I}$, QDs-Lec- ${ }^{125}$ I and Lec- ${ }^{125}$ I were examined on various cancer cell lines such as Caco-2, MCF-7 and A-549. The results showed more specificity of SNA towards MCF-7 cells compared with other cells lines. In addition, the QDsLec- ${ }^{131} \mathrm{I}$ used in in vivo studies indicated that the conjugated QDs were accumulated in the liver and bladder. These results suggested that the conjugate QDs-Lec- ${ }^{125 / 131} \mathrm{I}$ presented both radioactive and fluorescent properties could be a useful tool for tumour imaging and radiotherapy.

Kara et al. [53] prepared CdSe/CdS QDs, conjugated with PHA-L and labelled with ${ }^{125} \mathrm{I}$ to study the interface between phytohemagglutinin-L (PHA-L) and sialic acid. Sialic acid is abundant on the breast cancer cell (MCF-7) surface. The authors demonstrated that ${ }^{125} \mathrm{I}$ labeled QDPHA-L conjugates represent significant affinity on MCF-7 cells and the cell incorporation increased with time. This result was also confirmed by computational simulation using crystal structure of PHA-L, which revealed that the conjugates had a significant affinity for cells.

\subsection{Carbohydrate-functionalised QDs in cancer diagnosis and treatment}

Lack of specificity of formulated drug or nanomaterial is the major challenge for the effective cancer treatment. Therefore, the development of targeted system for cancer diagnosis and treatment is highly desirable. Cancer cell differ from its malignant counterparts, which provides valuable characteristic to develop specific targeted system for cancer. Targeted fluorescent biomarkers have found great advantage in the specific visualisation of cancer cells, which enables early-stage detection of cancers instead of 
depending on advanced morphological changes alone. Moreover, targeted system enhanced drug delivery to specific cancer cells or tissue thereby dramatically improving the selectivity and efficacy of anti-cancer drugs. Carbohydrates have attracted considerable attention in the development of targeting systems due to their ability to differentiate and recognise cells and the endocytic uptake resulting from specific carbohydrate-lectin interactions. Table 2 presents a summary of carbohydratefunctionaslied QDs targeting different cancer models for potential cancer treatment discussed in the text below.

\subsubsection{Carbohydrate-functionalised QDs for cancer imaging}

Ohyanagi et al. [55] investigated the dynamic distribution profiles of carbohydrate-functionalised QDs in model animals. The authors synthesised NIR fluorescent $\mathrm{CdSeTe} / \mathrm{CdS}$ QDs and coated them with a mixed population of thiol. The obtained QDs were further coated with different carbohydrate to form a collection of carbohydrate-functionalised QDs for in vivo study. NIR images of mice injected with carbohydrate-coated QDs exhibited significant differences in time-dependent distribution (Fig. 3). The authors demonstrated that sialyl $\mathrm{N}$-acetyllactosamine (sialylLacNAc) linked QDs accumulated in the spleen and intestine $2 \mathrm{~h}$ post injection, while LacNAc-functionalized QDs and Lex-QDs were localised in the liver, and no preferential distribution was observed for sLex-QDs. These results suggest that the structure of the carbohydrate residues in the individual sialylated oligosaccharides might influence significantly the organspecific distribution of the glycan functionalised QDs.

It has been well reported in the literature that hepatocytes, especially hepatocellular carcinoma cells (e.g., HepG2) have overexpressed galactose receptorsAsialoglycoprotein receptors (ASGP-Rs) [67-71]. Thus, ASGP-Rs could be an ideal target for liver cancer cells [70,71]. Bavireddia and Kikkeri [56] synthesised $\beta$-cyclodextrin $(\beta$-CD) capped with $O$ - $\alpha$-mannopyranoside (man) and $O-\beta$-galactopyranoside (gal) to $\mathrm{CdSe} / \mathrm{ZnS}-$ TOPO QDs. They first studied the interaction of synthesised nanoparticles with different lectins like ConA, Galanthus nivalis agglutinin (GNA) and peanut agglutinin (PNA) and observed that QDs- $\beta$-CD-man conjugates were successfully linked to ConA and GNA, while QDs- $\beta$-CD-gal conjugates only showed affinity with PNA. They later introduced these multivalent fluorescent nanoparticles in HepG2 cells and observed that only $\beta$-CDgal QDs internalized in liver carcinoma cells, which confirms Asialoglycoprotein receptor mediate interaction. Shinchi et al. [57] also proved similar concept by synthesizing $\beta$-galactose and $\alpha$-glucose $\mathrm{CdTe} / \mathrm{CdS} \mathrm{QDs}$ and studied there interaction with ConA and Ricinus communis agglutinin I (RCA 120). They proved that $\beta$-galactose $\mathrm{CdTe} / \mathrm{CdS}$ QDs internalised in HepG2 cells, which express asialoglycoprotein receptors, whereas $\alpha$-glucose QDs showed poor uptake in HepG2 liver carcinoma cells. They later then synthesised small library of cadmium free sugar ZnS-AgInS2 QDs such as $\alpha$-glucose, $\alpha$ - $N$-acetylglucose, $\beta$-galactose, mannose or

Table 2 Summary of carbohydrate-functionalised QDs targeting various types of cancer reported in literature

\begin{tabular}{|c|c|c|c|c|c|}
\hline Applications & Carbohydrate & Types of QDs & Targeting receptor & Cancer model & Ref. \\
\hline Cancer imaging & SialylLacNAc, LacNAc, Lex & PC-QDs & - & Mice model & {$[55]$} \\
\hline Cancer cell imaging & Mannose and galactose & CdSe/ZnS-TOPO QDs & $\begin{array}{l}\text { Asialoglycoprotein } \\
\text { receptors }\end{array}$ & HepG2 cells & {$[56]$} \\
\hline Cancer cell imaging & $\beta$-Galactose and $\alpha$-glucose & $\mathrm{CdTe} / \mathrm{CdS}$ QDs & $\begin{array}{l}\text { Asialoglycoprotein } \\
\text { receptors, glucose } \\
\text { receptor }\end{array}$ & HepG2 cells & {$[57]$} \\
\hline Cancer cell imaging & $\begin{array}{c}\alpha \text {-Glucose, } \alpha-N \text {-acetylglucose, } \\
\beta \text {-galactose, mannose or sialic } \\
\text { acid }\end{array}$ & ZnS-AgInS2 QDs & $\begin{array}{l}\text { Asialoglycoprotein } \\
\text { receptors }\end{array}$ & $\begin{array}{l}\text { Leukemia (THP-1), macrophage } \\
\text { (J774.A1) and HepG2 cells }\end{array}$ & {$[58]$} \\
\hline Cancer cell imaging & D-Mannose & Silicon QDs & - & MCF-7 cells & {$[59]$} \\
\hline Cancer cell imaging & Glucose, lactose & Silicon QDs & - & B16F10 melanoma cells & {$[60]$} \\
\hline Cancer cell imaging & Glucose & Silicon QDs & - & HeLa cells & {$[61]$} \\
\hline Cancer targeting & Galactose & $\mathrm{CdTe} / \mathrm{ZnS}-\mathrm{TOPO}$ & $\begin{array}{l}\text { Asialoglycoprotein } \\
\text { receptors }\end{array}$ & HeLa and A549 cells & {$[62]$} \\
\hline Cancer targeting & D-Mannose & Silicon QDs & - & MCF-7 cells & {$[63]$} \\
\hline Cancer targeting & $\begin{array}{l}\text { Galactose, glucose, mannose, } \\
\text { and lactose }\end{array}$ & Silicon QDs & - & $\begin{array}{l}\text { MCF-7, HepG2, A549, SK-Mel, } \\
\text { HHL5, HeLa cells }\end{array}$ & {$[64]$} \\
\hline Cancer targeting & Galactose & CdSe-ZnS QDs & Galactose receptors & HepG2 and HeLa cells & {$[65]$} \\
\hline Cancer theranostics & Mannose & Albumin-CdTe QDs & - & $\begin{array}{c}\text { MCF-7 and MDA-MB-231 cells, } \\
\text { Ehrlich ascites tumour in BALB/C } \\
\text { mice }\end{array}$ & {$[66]$} \\
\hline
\end{tabular}




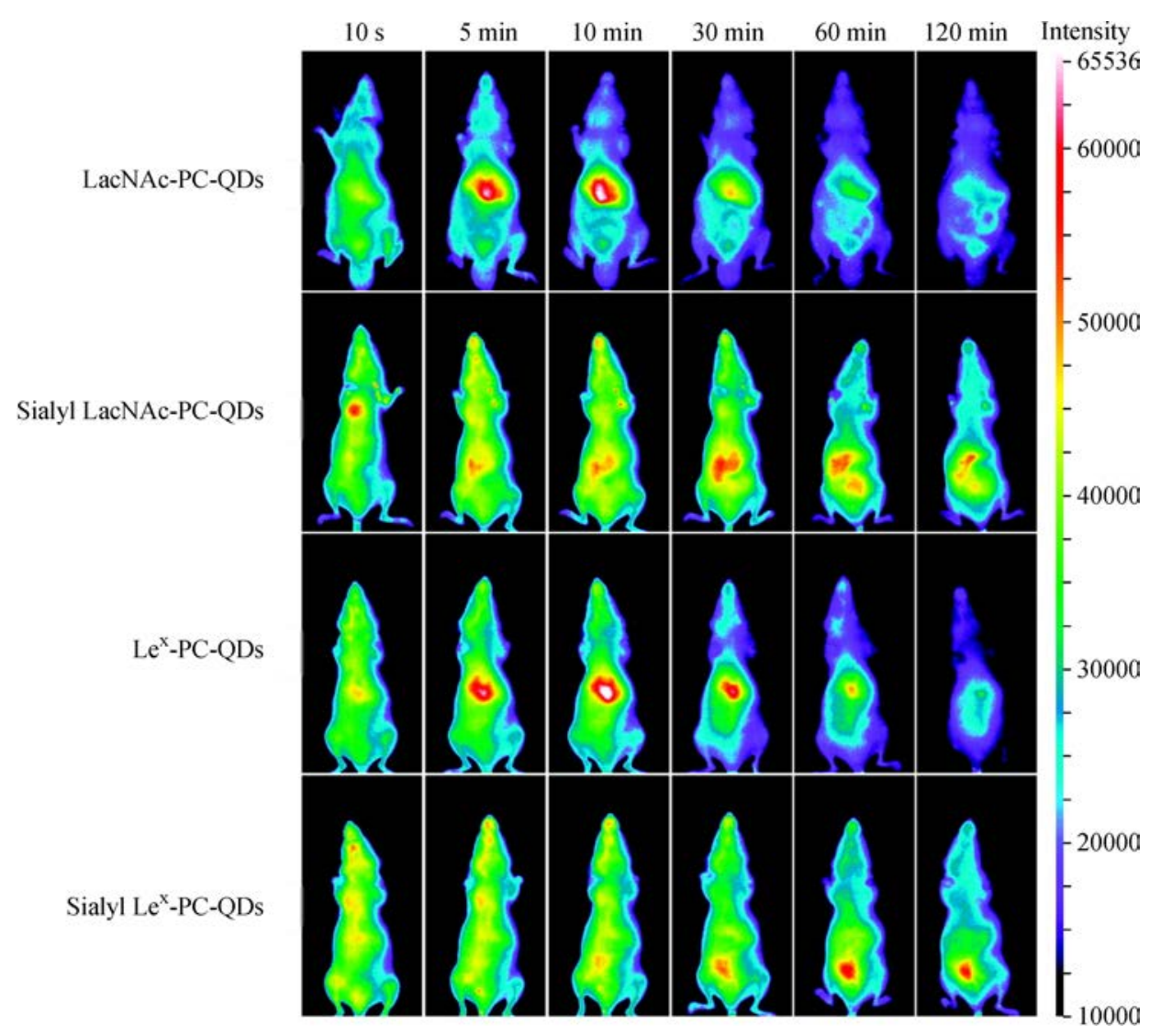

Fig. 3 Live animal imaging module of mice treated with glyco-functionalised QDs carrying Lewis antigen-related oligosaccharides. (Reproduced with permission from Ref. [55], Copyright American Chemical Society)

sialic acid [58]. They used three different cell lines, leukaemia (THP-1), macrophage (J774.A1) and HepG2 cells. The results showed that leukaemia cells only uptaken $\alpha$-glucose QDs, perhaps due to the lack of carbohydrate receptors. All the QDs were internalised in Macrophage cells, whereas HepG2 cells were preferentially taken up $\beta$ galactose QDs, due to the high amount of asialoglycoprotein receptors in these cells' membranes.

Zhai et al. [59] have also developed a straightforward synthesis method for carbohydrate-functionalised silicon quantum dots (SiQDs). The authors used three surface modifications to study SiQDs solubility in water and their biocompatibility within cells: L-alanine and pentanoic acid were chosen for their structural simplicity and D-mannose surface functionalities were used for targeted imaging of MCF-7 breast cancer cells. Fluorescence microscopy demonstrated the D-mannose and L-alanine functionalised SiQDs could readily internalised by MCF-7 cells, however, the pentanoic acid terminated SiQD did not. This observation indicates carbohydrate-functionalised SiQDs can be effective luminescent imaging agents.

Lai et al. [60] created structurally defined and fluorescently labelled multifunctional carbohydrate-capped silicon nanoparticles (SiNPs) to study ultra-weak carbohydrate-carbohydrate interactions by surface plas- mon resonance (SPR) and cell imaging. An additional dye (ATTO647N) was added to create dual-fluorescent SiNPs. They synthesised three different sugar-capped SiNPs (Glcdye@SiNP, Lac-dye@SiNP, and Gg3-dye@SiNP) and subsequently used to determine the low-affinity interaction of the two glycosphingolipids GM3 and Gg3. Specific binding of Gg3-dye@SiNPs to immobilized GM3-biotinPAA was detected, and affinity analysis was performed by SPR confirming the existence of this carbohydratecarbohydrate interaction. Moreover, they demonstrated that sugar-dye@SiNPs are valuable tools for cell imaging by the uptake of Gg3-dye@SiNPs into GM3-expressing B16F10 melanoma cells (Fig. 4).

Hsu et al. [61] also synthesized ultra-small SiNPs (i.e., SiQDs) for cancer cell imaging purpose. The SiQDs were functionalised with glucose moieties on their surface (SiGlc). The amount of glucose on the surface of SiQDs could be well controlled by varying the ratio between the amount of SiQDs and the saccharide groups during the coupling reaction. The photophysical behaviour of the glucose functionalised SiQDs were not significantly different to that of the unmodified nanoparticles. In vitro studies performed on HeLa cells demonstrated faster internalisation $(1 \mathrm{~h})$ of Si-Glc into the cells compared to the unmodified NPs (24 h). Furthermore, CASY assay showed 


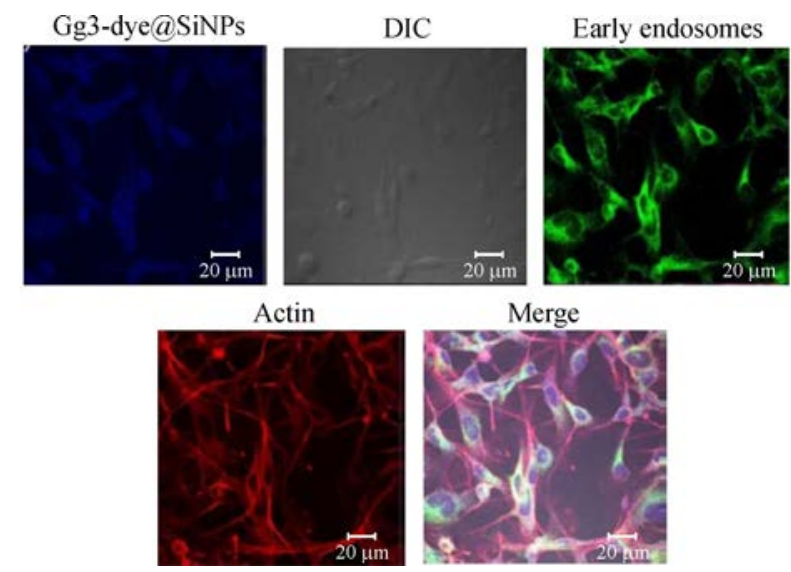

Fig. 4 Confocal fluorescent images showing the uptake of Gg3dye@SiNPs by B16F10 cells after 2 h incubation. SiNPs inherent fluorescence-blue, DIC-differential interference contrast microscopy, early endosomes-green (stained with an anti-EEA1 antibody), actin cytoskeleton-red (stained with Alexa 555-labeled phalloidin). (Adapted with permission from Ref. [60], Copyright American Chemical Society)

that Si-Glc exhibited high cell viability. These findings suggested these glucose functionalised SiQDs could be potentially interesting alternatives as cancer bioimaging probes.

\subsubsection{Carbohydrate-functionalised QDs for cancer targeting} and theranostics

Cheng et al. [62] developed hydrophilic L-cysteine coated CdSeTe QDs conjugated with Con A, and then with $\mathrm{N}$-acetylglucosamine (GlcNAc). The QDs-ConA-GlcNAc bioconjugate was formed by lectin-carbohydrate interaction between Con A and GlcNAc. GlcNAc possesses a strong affinity for Hsp70 (70 kDa Heat Shock Protein), an important protein involved in tumour cell proliferation and may be a potential biomarker for cancer cells [62,72-74]. The obtained bioconjugates has an emission wavelength at $650 \mathrm{~nm}$ and displayed a high fluorescence intensity with specific binding to HeLa cells (cervical cancer cells). The co-localization of red fluorescence from QDs-Con A-GlcNAc bioconjugate with the green fluorescence from FITC-immunoglobulin $G$ suggested that these Glyo-QDs can target the Hsp70 protein. These results show that the QDs-Con A-GlcNAc bioconjugate can be a promising tool for direct localisation of the Hsp70 protein, therefore recognise cancers related to this protein.

Ahire et al. [63] developed D-mannose capped SiNPs for targeting MCF-7 breast cancer cells. Visualisation imaging of SiNPs in MCF-7 human breast cancer cells showed the fluorescence was distributed throughout the cytoplasm of these cells. Later the same research group prepared SiNPs capped with different carbohydrates including galactose, glucose, mannose, and lactose from amine terminated SiNPs $[64,75]$. The MTT analysis showed an extensive reduction in toxicity of SiNPs by functionalizing with carbohydrate moiety in vitro. In vivo toxicity assay on the $X$. laevis embryos showed that carbohydrate capped SiNPs do not induce severe toxicity, however the amine terminated SiNPs caused the death of the embryos (Fig. 5(A)). They reported that the carbohydrate-functionalised SiNPs internalized in the cells within $24 \mathrm{~h}$ of incubation and reached the optimal concentration within the cells (Fig. 5(B)). Furthermore, they showed a reduced internalization of the carbohydrate-functionalised SiNPs at $4^{\circ} \mathrm{C}$ compared to $37^{\circ} \mathrm{C}$, which suggested the cellular uptake of the carbohydrate capped SiNPs is likely to be receptor-mediated and energy dependent. They also showed that the up-taken of the carbohydrate capped SiNPs were more readily by cancer cells (A549, MCF-7 and SK-Mel28) than non-cancerous cells (MDCK and HHL5, Fig. 5(C)). Moreover, they demonstrated the use of carbohydrates for the internalisation of a variety of similar compounds into cancer cells.

It is well known that galactose receptors are overexpressed in certain types of cancer cells, for example hepatocellular carcinoma [70,71]. However, the role of galactose multivalency on cellular processes are largely unexplored. To study these, Dalal and Jana [65] have synthesised galactose functionalised multivalent QDs with the average numbers of galactose per QD of 25, 50, and 80 [QD(gal)25, QD(gal)50, and QD(gal)80]. The uptake mechanism of the multivalent QDs was investigated in galactose receptor overexpressed HepG2 cells. The authors have demonstrated that the cellular interaction and uptake kinetics of these galactose functionalised QDs increased with increasing galactose multivalency, while the uptake mechanism shifts from lipid raft/caveolae-mediated endocytosis to clathrin-mediated endocytosis as galactose multivalency increased. They have also found that lower multivalent QDs reside in the cytoplasm for a longer time compared to higher multivalent QDs, but their endosomal/ lysozomal trapping and exocytosis increased as increasing galactose multivalency. The demonstrated finding is agreed with the extensive study done by Zhang and Monteiro-Riviere [76]. They have demonstrated that the uptake of un-conjugated QDs probably regulated via lipid raft/caveolae-mediated endocytosis. They also showed that after internalisation into the cytoplasm, the QDs entered early endosomes and then transferred into late endosomes or lysosomes. In addition, they showed that QD endocytic pathway is primarily regulated by the G-protein coupled receptor associated pathway. These findings demonstrate the functional role of galactose multivalency on cellular interaction, cell uptake mechanism, and subcellular targeting, which could be proposed for subcellular targeting applications.

By combining the carbohydrate specific targeting towards cancer cells and strong fluorescence imaging capability of QDs, Zayed et al. [66] have developed a smart cancer nano-theranostics system. The authors attached 
(A)
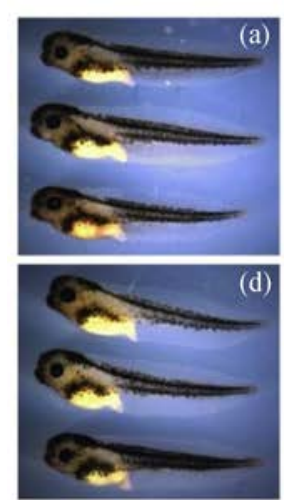

Glu SiNPs

$200 \mu \mathrm{g} / \mathrm{mL}$
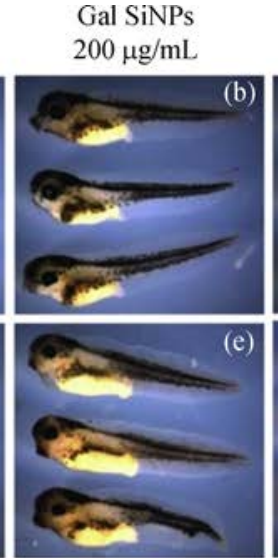

Lac SiNPs $200 \mu \mathrm{g} / \mathrm{mL}$
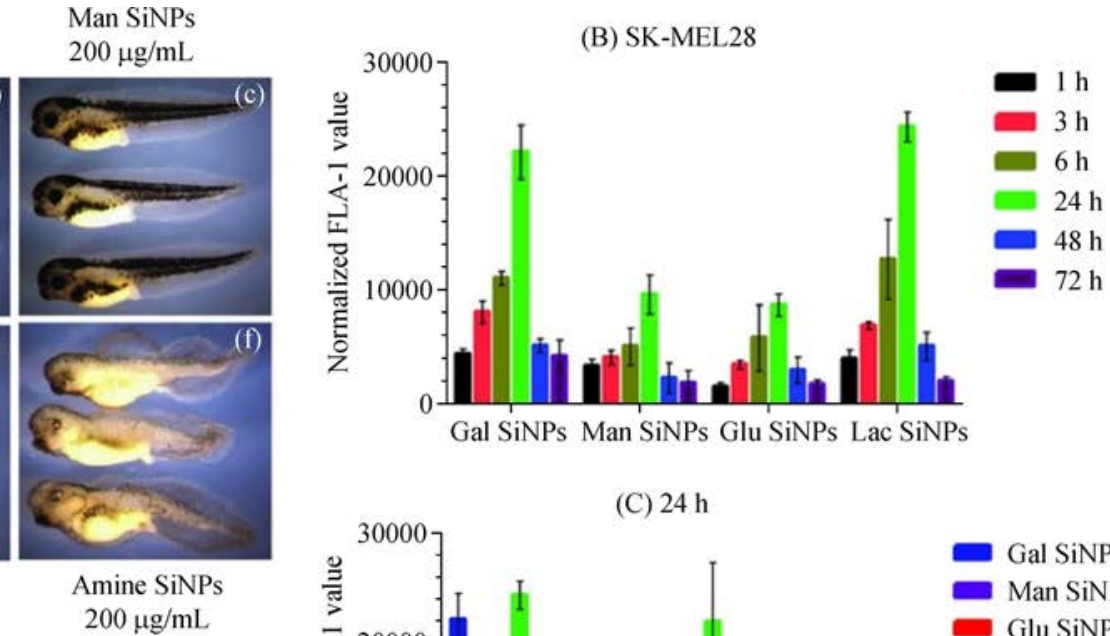

(C) $24 \mathrm{~h}$

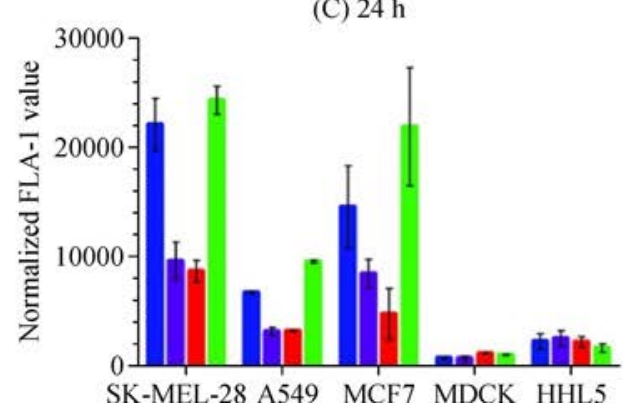

Fig. 5 (A) In vivo toxicity assay using X. laevis embryos. Embryos exposed to carbohydrate capped SiNPs at a concentration of 200 $\mu \mathrm{g} \cdot \mathrm{mL}^{-1}$ : (a) control, (b) gal capped SiNPs, (c) man capped SiNPs, (d) Glu capped SiNPs, (e) Lac capped SiNPs, and (f) amine-terminated SiNPs. Embryos were exposed to the SiNPs at NF stage 15 and scored at NF stage 38; (B) Time dependent uptake efficiency of carbohydrate capped SiNPs in SK-Mel28 cells at various incubation times; (C) Uptake efficiency of carbohydrate capped SiNPs in cancer cells (A549, SK-Mel28, and MCF-7) and noncancerous cells (MDCK, HHL5) at 24 h. (Copyright Wiley, adapted with permission from Ref. [64])

both biocompatible albumin backbone and mannose moieties to CdTe QDs for enhanced tumour targeting and reduced QDs toxicity. They have then used a combination therapy that co-loading anticancer drug pemetrexed and resveratrol to the QD platform for tumour site-specific release. The mannose functionalised albumin-QDs theranostics could be tracked by their high fluorescence quantum yield and showed enhanced cytotoxicity and internalization into MCF-7 and MDA-MB-231 breast cancer cells. Moreover, in vivo bioimaging demonstrated excellent tumour-specific accumulation of the mannosegrafted theranostic QDs. The theranostic QDs also showed effective anti-tumour activities including reduced tumour volume, increased apoptosis, and inhibited angiogenesis. Overall, mannose-grafted theragnostic QDs nanoplatform could be a potential nano-theranostic for bioimaging and targeted breast cancer therapy.

\section{Challenges faced in using glyco-functio- nalised QDs in biomedical applications}

\subsection{Nanotoxicology}

Despite of QDs showing great potential for biomedical imaging and detection, limitation of its application arises mainly from heavy metal, colloidal instability and oxidation of the nanoparticle core/shell material, which constraint the improvement towards the diagnosis and therapy of cancer. These concerns serve as great barrier for human application in vivo cancer imaging than the development of the application in vitro. Huge efforts have been made to generate novel QDs that minimize toxicity and maximize detection efficiency by changing their components such as sizes, surface coatings, and valences etc. Nanoparticles coating is a promising approach to reduce the cytotoxicity because these additional layers act as a physical barrier to the core/shell to prevent oxidation. Ligand shows a negative charge at biological $\mathrm{pH}$ have been widely used for solubilisation of QDs including carboxylic acid, hydroxyl or amine groups. PEG coated QD has been proven to be remarkably stable and almost completely removed cytotoxicity. However, such surface modification reveals reduced uptake by cells compared to other surface coatings. It is important to know that layers of coating should not only protect the QD core/ shell from oxidation but must also proliferate the cellular uptake. Simply adding more layers to achieve desirable results is not a favourable strategy for biological applications, because it will largely increase the size of the QDs and become an issue.

Carbohydrate-functionalised nanomaterials showing 
outstanding biocompatibility, biodegradability, high diversity of chemical functionalities, and versatile biological functions in biomedical applications. The study of glyconanomaterials, on the other hand, is a new field and the progress has been relatively covering. The vast majority of glyconanomaterial are made from simple, low-cost carbohydrate structure such as monosaccharides. The conjugation process is mostly general chemistry and can be useful to attach these carbohydrates to an extensive diversity of nanomaterials. Glyconanomaterial act as a multivalent scaffold carrying multiple copies of carbohydrate molecules, thus elaborating the binding affinity with the recognition receptors. It has been reported that carbohydrate-functionalised QDs show no/minimal cytotoxicity both in vivo and in vitro environment. This carbohydrate functionality not only act as physical barrier to provide stability but also serves a specific targeting ligand.

Nevertheless, few additional objectives should also be considered such as coating shell degradation caused by modification of QDs, nonspecific accumulation by liver, spleen and lymphatic system, immune response and genotoxic effect etc. Considering the toxicity of $\mathrm{Cd}$, Se, $\mathrm{Zn}, \mathrm{Te}, \mathrm{Hg}$, and $\mathrm{Pb}$, several low toxicity QDs have been established as substitutes. For example, carbon dots less than $10 \mathrm{~nm}$ appear to be an ideal alternative to $\mathrm{Cd} 2 / \mathrm{Pb}$ based semiconductor QDs for their tunable stable fluorescence emission, low cost, and low toxicity [77]. The same group have also synthesised graphene quantum dots for direct and efficient stem cell labelling [78] and cancer diagnosis [79]. Non-toxic elements doping could be another way to achieve low toxicity. These doped QDs have tunable fluorescence and high quantum efficiency and are ideal candidates for biomedical applications. Silicon QDs are exceptional candidate and have been showing promising results in reduced cytotoxicity $[75,80]$. For in vivo applications, Silicon NPs provide attractive chemical alteration to heavy-metal containing QDs, which are shown to be toxic in biological environments. In addition silicon is a common trace element in human body, it is reported that silicic acid administrated to human is efficiently excreted from the body through urine [81]. It can be expected that multifunctional, non-toxic silicon nanostructure in future may provide promising application in clinical translation. Nevertheless, before employing QDs in any medical procedure extensive analysis and research on the toxicity profiles will be needed. Further studies are also needed to investigate the long-term toxicology and pharmacokinetics of QDs from living systems involving degradation, clearance, persistence, and immune response of QDs.

3.2 Design and generation of biocompatible and biodegradable glyco-functionalised QDs

The current limitation of QDs in vivo imaging is due to the non-specific organ uptake and reticuloendothelial system (RES) scavenging, which are mainly because of large size, colloidal instability and short circulation half-life in the blood vascular system. A number of literature demonstrated the fate of QDs in vivo has been affected by particle size and surface functionality [82-84]. Choi et al. [85] have defined the requirements for renal filtration and urinary excretion of QD using rodents as a model system. They have also suggested to use zwitterionic or neutral organic coatings to prevent adsorption of serum proteins, which otherwise increased QD's hydrodynamic diameter and prevent renal excretion. The author concluded that the size of QD should be controlled under $5.5 \mathrm{~nm}$ to achieve efficient urinary excretion and elimination of QDs. These findings have also been confirmed by other group, for example Zhu et al. [86] showed that the QD size less than $5 \mathrm{~nm}$ was mainly found in the bladder at 4 hours after intravenous injection, while QDs larger than $5 \mathrm{~nm}$ accumulated primarily in the lung, spleen, and liver, indicating a different excretion pattern.

Continuous efforts are attempted to prevent adsorption of QDs to the plasma proteins and prolong their circulation time by coating them with passivating molecules. The multivalent effect of glyconanomaterial increases the binding affinity with the recognition receptors, thus it is considered as simple glycan-presenting cell/virus-mimics able to interact with other biological entities. Compared to free, unbound carbohydrates, glyco-functionalised QDs exhibit several orders of magnitude higher binding affinity with lectins. However, the multivalent effect is dependent on several factors including conjugation chemistry, spacer linkage, ligand density and spatial arrangement. In order to produce effective glyco functionalised QDs for biomedical application, comprehensive understanding of all these issues is needed.

The unique physical properties of nanomaterial and recognition ability of carbohydrate ligands provide benefit to glyconanomaterial in biomedical application. Carbohydrate ligands selectively interact with the receptors on cell surface, and trigger binding, cell agglomeration, or particle internalisation. Altogether with glyco functionalisation and unique physical properties of nanomaterial advance to translate molecular events into noticeable or clear signals allowing for imaging and revealing of disease state. These glyconanoparticles have been used in fluorescence, magnetic resonance imaging (MRI) and positron emission tomography-computed tomography (PET/CT) imaging to distinguish normal and cancer cell lines or to detect tumours. Glyconanomaterials have demonstrated promising results in biocompatibility. Recent studies by VelaRamirez et al. [87] reported the safety and biocompatibility of carbohydrate-functionalized polyanhydride nanoparticles upon parenteral and intranasal administration. The results showed that a 5-mg dose of either linker- or di-mannose-functionalised nanoparticles did not induce hepatic or renal tissue damage or cause elevation of 
damage-related or functional biomarkers in serum or urine following subcutaneous administration.

3.3 Reproducibility, reliability, and comparability of glycofunctionalised QDs

The major limitation of QDs when using in clinical application is their reproducibility and comparability as well as their potential for quantification. Glyco-functionalised QDs are showing promising results for biomedical applications however there is insufficient amount of data on their reproducibility and comparability. Different functionalisation from various carbohydrate will results in different quantum yield based on various material and surface chemistries. Moreover, the absolute control on quantification of carbohydrate moiety on the surface of QDs is also in need when using surface chemistry. Purity is also one of the concerns when applying glyco-functionalised QDs in clinical trial. Thus, the origin and establishment of quality standards for these advance materials of various carbohydrate-functionalised QDs is the essential initial step in targeting cancer.

\section{Future perspective}

In the near future the research of glycol-conjugated QDs in cancer imaging will significantly improve their clinical application in targeting metastasis and in quantitative measurement of molecular targets. The ongoing development of glyco-functionalised QDs can target solid tumour tissues with mature vasculature, however it is challenged to target micro-metastasis without well-developed vasculature. Therefore, the surface of glyco functionalised QDs needs to be further engineered to enable efficient extravasation, to reach micro-metastasis and initiate binding to tumour antigens. The progressive research in glyco-conjugated QDs will improve early cancer detection and their clinical application in targeting metastasis tumour in near future. The ongoing development of glycofunctionalised QDs can target solid tumour tissues, however it is still challenged to target micro-metastatic tumour. Therefore, it is important to further engineered the carbohydrate moiety's to target specific micro-metastasis tumour and initiate binding with antigens. It is also important to minimize the RES uptake and maximize the tumour specific uptake by engineering tumour specific targeting carbohydrate moiety on the surface of QDs. Because metastasis is possible in RES system, especially in liver and lymph node, non-specific RES uptake will result in false-positive results. Long circulation time in blood and colloidal stability is also another important aspect; moreover, it also helps in reducing RES uptake. Glycosilyated PEG proves to be good candidate for longer circulation in blood and minimizing RES uptake nonetheless finally size can become issue. When using for biomedical application or cancer therapy, systemic toxicity is an important feature. QD toxicity is well known and it is still a topic of concern, however it has shown that carbohydrate functionality helps reducing nanoparticle toxicity. Perhaps silicon QDs will emerge as a striking substitute to heavy metal-containing QDs, due to lower toxicity potential, and progress further biological and clinical applications. Optimal clearance of modified QDs from body is additional issue, which needs to be address; toxicity is also induced by poor clearance from body. Thus, it would be viable to design and create such promising therapeutic agent with more favourable clearance properties.

\section{Conclusions}

Glyconanotechnology's emerging progress in cancer therapy, diagnosis and theranostic applications have revolved these nanoprobes very attractive branch of the world scientific research. Combining these tools along with unique fluorescent characteristic of QDs and specificity of carbohydrate functionality enables real-time studies, introduces new opportunities for several biomedical applications. More theranostic applications could be developed by selecting and engineering specific tumour carbohydrate functionality along with unique featured QDs.

Open Access This article is licensed under a Creative Commons Attribution 4.0 International License, which permits use, sharing, adaptation, distribution and reproduction in any medium or format, as long as you give appropriate credit to the original author(s) and the source, provide a link to the Creative Commons licence, and indicate if changes were made. The images or other third party material in this article are included in the article's Creative Commons licence, unless indicated otherwise in a credit line to the material. If material is not included in the article's Creative Commons licence and your intended use is not permitted by statutory regulation or exceeds the permitted use, you will need to obtain permission directly from the copyright holder. To view a copy of this licence, visit http://creativecommons.org/licenses/by/4.0/.

\section{References}

1. Bentolila L A, Ebenstein Y, Weiss S. Quantum dots for in vivo small-animal imaging. Journal of Nuclear Medicine: Official Publication, Society of Nuclear Medicine, 2009, 50(4): 493-496

2. Byers R J, Hitchman E R. Quantum dots brighten biological imaging. Progress in Histochemistry and Cytochemistry, 2011, 45 (4): 201-237

3. Tholouli E, Sweeney E, Barrow E, Clay V, Hoyland J, Byers R. Quantum dots light up pathology. Journal of Pathology, 2008, 216 (3): 275-285

4. He X, Gao J, Gambhir S S, Cheng Z. Near-infrared fluorescent nanoprobes for cancer molecular imaging: Status and challenges. Trends in Molecular Medicine, 2010, 16(12): 574-583

5. Hilderbrand S A, Weissleder R. Near-infrared fluorescence: Application to in vivo molecular imaging. Current Opinion in Chemical Biology, 2010, 14(1): 71-79 
6. Wang Y, Chen L. Quantum dots, lighting up the research and development of nanomedicine. Nanomedicine (London), 2011, 7 (4): 385-402

7. Varki A, Cummings R D, Esko J D, Freeze H H, Stanley P, Bertozzi C R, Hart G W, Etzler M E. Essentials of Glycobiology. 3rd ed. New York: Cold Spring Harbor Laboratory Press, 2009

8. Calvaresi E C, Hergenrother P J. Glucose conjugation for the specific targeting and treatment of cancer. Chemical Science (Cambridge), 2013, 4(6): 2319-2333

9. Kottari N, Chabre Y M, Sharma R, Roy R. Applications of glyconanoparticles as "sweet" glycobiological therapeutics and diagnostics. In: Multifaceted Development and Application of Biopolymers for Biology, Biomedicine and Nanotechnology. Dutta P K, Dutta J, eds. Berlin: Springer International Publishing, 2013

10. Marradi M, Chiodo F, Garcia I, Penades S. Glyconanoparticles as multifunctional and multimodal carbohydrate systems. Chemical Society Reviews, 2013, 42(11): 4728-4745

11. Luczkowiak J, Munoz A, Sanchez-Navarro M, Ribeiro-Viana R, Ginieis A, Illescas B M, Martin N, Delgado R, Rojo J. Glycofullerenes inhibit viral infection. Biomacromolecules, 2013, 14(2): 431-437

12. Ribeiro-Viana R, Sánchez-Navarro M, Luczkowiak J, Koeppe J R, Delgado R, Rojo J, Davis B G. Virus-like glycodendrinanoparticles displaying quasi-equivalent nested polyvalency upon glycoprotein platforms potently block viral infection. Nature Communications, 2012, 3(1): 1303

13. Fasting C, Schalley C A, Weber M, Seitz O, Hecht S, Koksch B, Dernedde J, Graf C, Knapp E W, Haag R. Multivalency as a chemical organization and action principle. Angewandte Chemie International Edition, 2012, 51(42): 10472-10498

14. Liu B, Lu X, Ruan H, Cui J, Li H. Synthesis and applications of glyconanoparticles. Current Organic Chemistry, 2016, 20(14): 1502-1511

15. Reichardt N C, Martin-Lomas M, Penades S. Glyconanotechnology. Chemical Society Reviews, 2013, 42(10): 4358-4376

16. Sharon N, Lis H. History of lectins: From hemagglutinins to biological recognition molecules. Glycobiology, 2004, 14(11): 53R-62R

17. Sharon N, Lis H. Lectins as cell recognition molecules. Science, 1989, 246(4927): 227-234

18. Ashwell G, Harford J. Carbohydrate-specific receptors of the liver. Annual Review of Biochemistry, 1982, 51(1): 531-554

19. Belardi B, Bertozzi C R. Chemical lectinology: Tools for probing the ligands and dynamics of mammalian lectins in vivo. Chemistry \& Biology, 2015, 22(8): 983-993

20. André S, Kaltner H, Manning J C, Murphy P V, Gabius H J. Lectins: Getting familiar with translators of the sugar code. Molecules (Basel, Switzerland), 2015, 20(2): 1788-1823

21. Surolia A, Bachhawat B K, Podder S K. Interaction between lectin from ricinus communis and liposomes containing gangliosides. Nature, 1975, 257(5529): 802-804

22. Häuselmann I, Borsig L. Altered tumor-cell glycosylation promotes metastasis. Frontiers in Oncology, 2014, 4: 28

23. Friedel M, Andre S, Goldschmidt H, Gabius H J, Schwartz-Albiez R. Galectin-8 enhances adhesion of multiple myeloma cells to vascular endothelium and is an adverse prognostic factor.
Glycobiology, 2016, 26(10): 1048-1058

24. Compagno D, Gentilini L D, Jaworski F M, Pérez I G, Contrufo G, Laderach D J. Glycans and galectins in prostate cancer biology, angiogenesis and metastasis. Glycobiology, 2014, 24(10): 899-906

25. Vazquez-Levin M H, Marin-Briggiler C I, Caballero J N, Veiga M F. Epithelial and neural cadherin expression in the mammalian reproductive tract and gametes and their participation in fertilization-related events. Developmental Biology, 2015, 401(1): 2-16

26. Ng K, Ferreyra J, Higginbottom S, Lynch J, Kashyap P, Gopinath S, Naidu N, Choudhury B, Weimer B, Monack D, Sonnenburg J L. Microbiota-liberated host sugars facilitate post-antibiotic expansion of enteric pathogens. Nature, 2013, 502(7469): 96-99

27. Becer C R. The glycopolymer code: Synthesis of glycopolymers and multivalent carbohydrate-lectin interactions. Macromolecular Rapid Communications, 2012, 33(9): 742-752

28. Kazunori M, Miki H, Takayasu I, Yoshinao Y, Kazukiyo K. Selforganized glycoclusters along DNA: Effect of the spatial arrangement of galactoside residues on cooperative lectin recognition. Chemistry (Weinheim an der Bergstrasse, Germany), 2004, 10(2): 352-359

29. Brus L E. Electron-electron and electron-hole interactions in small semiconductor crystallites: The size dependence of the lowest excited electronic state. Journal of Chemical Physics, 1984, 80(9): 4403-4409

30. Alivisatos A P, Gu W, Larabell C. Quantum dots as cellular probes. Annual Review of Biomedical Engineering, 2005, 7(1): 55-76

31. Foote M. The importance of planned dose of chemotherapy on time: Do we need to change our clinical practice? Oncologist, 1998, 3(5): 365-368

32. Naumov G, Akslen L, Folkman J. Role of angiogenesis in human tumor dormancy: Animal models of the angiogenic switch. Cell Cycle (Georgetown, Tex.), 2006, 5(16): 1779-1787

33. Frangioni $J V$. New technologies for human cancer imaging. Journal of Clinical Oncology, 2008, 26(24): 4012-4021

34. Liu J, Levine A L, Mattoon J S, Yamaguchi M, Lee R J, Pan X, Rosol T J. Nanoparticles as image enhancing agents for ultrasonography. Physics in Medicine and Biology, 2006, 51(9): 2179-2189

35. Massoud T F, Gambhir S S. Molecular imaging in living subjects: Seeing fundamental biological processes in a new light. Genes \& Development, 2003, 17(5): 545-580

36. Albrecht T, Blomley M J K, Burns P N, Wilson S, Harvey C J, Leen E, Claudon M, Calliada F, Correas J M, LaFortune M, et al. Improved detection of hepatic metastases with pulse-inversion US during the liver-specific phase of SHU 508A: Multicenter study. Radiology, 2003, 227(2): 361-370

37. Blomley M J, Cooke J C, Unger E C, Monaghan M J, Cosgrove D O. Microbubble contrast agents: A new era in ultrasound. BMJ (Clinical Research Ed.), 2001, 322(7296): 1222-1225

38. Cormode D P, Skajaa T, Fayad Z A, Mulder W J. Nanotechnology in medical imaging: Probe design and applications. Arteriosclerosis, Thrombosis, and Vascular Biology, 2009, 29(7): 992-1000

39. Weissleder R. Scaling down imaging: Molecular mapping of cancer in mice. Nature Reviews. Cancer, 2002, 2(1): 8-11

40. Caravan P, Ellison J J, McMurry T J, Lauffer R B. Gadolinium(iii) chelates as MRI contrast agents: Structure, dynamics, and applications. Chemical Reviews, 1999, 99(9): 2293-2352 
41. Hoult D I, Phil D. Sensitivity and power deposition in a high-field imaging experiment. Journal of Magnetic Resonance Imaging, 2000, 12(1): 46-67

42. Jongmin S, Md A R, Kyeong K M, Ho I G, Hee L J, Su L I. Hollow manganese oxide nanoparticles as multifunctional agents for magnetic resonance imaging and drug delivery. Angewandte Chemie International Edition, 2009, 48(2): 321-324

43. Smith A M, Duan H, Mohs A M, Nie S. Bioconjugated quantum dots for in vivo molecular and cellular imaging. Advanced Drug Delivery Reviews, 2008, 60(11): 1226-1240

44. Zhang H, Yee D, Wang C. Quantum dots for cancer diagnosis and therapy: Biological and clinical perspectives. Nanomedicine (London), 2008, 3(1): 83-91

45. Wang H, Li H, Zhang W, Wei L M, Yu H X, Yang P Y. Multiplex profiling of glycoproteins using a novel bead-based lectin array. Proteomics, 2014, 14(1): 78-86

46. Munkley J, Elliott D J. Hallmarks of glycosylation in cancer. Oncotarget, 2016, 7(23): 35478-35489

47. Liu X, Nie H, Zhang Y B, Yao Y F, Maitikabili A, Qu Y P, Shi S L, Chen C Y, Li Y. Cell surface-specific $N$-glycan profiling in breast cancer. PLoS One, 2013, 8(8): 11

48. Scott E, Munkley J. Glycans as biomarkers in prostate cancer. International Journal of Molecular Sciences, 2019, 20(6): 20

49. Andrade C G, Cabral Filho P E, Tenório D P L, Santos B S, Beltrão E I C, Fontes A, Carvalho L B. Evaluation of glycophenotype in breast cancer by quantum dot-lectin histochemistry. International Journal of Nanomedicine, 2013, 8: 4623-4629

50. He D, Wang D, Shi X, Quan W, Xiong R, Yu C, Huang H. Simultaneous fluorescence analysis of the different carbohydrates expressed on living cell surfaces using functionalized quantum dots. RSC Advances, 2017, 7(20): 12374-12381

51. Cunha C R A, Andrade C G, Pereira M I A, Cabral Filho P E, Carvalho L B Jr, Coelho L C B B, Santos B S, Fontes A, Correia M T S. Quantum dot-cramoll lectin as novel conjugates to glycobiology. Journal of Photochemistry and Photobiology. B, Biology, 2018, 178: 85-91

52. Akca O, Unak P, Medine E I, Sakarya S, Yurt Kilcar A, Ichedef C, Bekis R, Timur S. Radioiodine labeled $\mathrm{CdSe} / \mathrm{CdS}$ quantum dots: Lectin targeted dual probes. Radiochimica Acta, 2014, 102(9): 849

53. Kara A, Ünak P, Selçuki C, Akça Ö, Medine E İ, Sakarya S. PHA-L lectin and carbohydrate relationship: Conjugation with $\mathrm{CdSe} / \mathrm{CdS}$ nanoparticles, radiolabeling and in vitro affinities on MCF-7 cells. Journal of Radioanalytical and Nuclear Chemistry, 2014, 299(1): 807-813

54. Santos B, de Farias P, de Menezes F, de Ferreira R, Júnior S, Figueiredo R, de Carvalho L, Beltrão E I C. CdS-Cd(OH $)_{2}$ core shell quantum dots functionalized with concanavalin a lectin for recognition of mammary tumors. Physica Status Solidi. C, Current Topics in Solid State Physics, 2006, 3(11): 4017-4022

55. Ohyanagi T, Nagahori N, Shimawaki K, Hinou H, Yamashita T, Sasaki A, Jin T, Iwanaga T, Kinjo M, Nishimura S I. Importance of sialic acid residues illuminated by live animal imaging using phosphorylcholine self-assembled monolayer-coated quantum dots. Journal of the American Chemical Society, 2011, 133(32): 1250712517

56. Bavireddi H, Kikkeri R. Glyco- $\beta$-cyclodextrin capped quantum dots: Synthesis, cytotoxicity and optical detection of carbohydrateprotein interactions. Analyst (London), 2012, 137(21): 5123-5127

57. Shinchi H, Wakao M, Nakagawa S, Mochizuki E, Kuwabata S, Suda Y. Stable sugar-chain-immobilized fluorescent nanoparticles for probing lectin and cells. Chemistry, an Asian Journal, 2012, 7(11): 2678-2682

58. Shinchi H, Wakao M, Nagata N, Sakamoto M, Mochizuki E, Uematsu T, Kuwabata S, Suda Y. Cadmium-free sugar-chainimmobilized fluorescent nanoparticles containing low-toxicity $\mathrm{ZnS}$ $\mathrm{AgInS}_{2}$ cores for probing lectin and cells. Bioconjugate Chemistry, 2014, 25(2): 286-295

59. Zhai Y, Dasog M, Snitynsky R B, Purkait T K, Aghajamali M, Hahn A H, Sturdy C B, Lowary T L, Veinot J G C. Water-soluble photoluminescent D-mannose and L-alanine functionalized silicon nanocrystals and their application to cancer cell imaging. Journal of Materials Chemistry. B, Materials for Biology and Medicine, 2014, 2(47): 8427-8433

60. Lai C H, Hütter J, Hsu C W, Tanaka H, Varela-Aramburu S, De Cola L, Lepenies B, Seeberger P H. Analysis of carbohydratecarbohydrate interactions using sugar-functionalized silicon nanoparticles for cell imaging. Nano Letters, 2016, 16(1): 807-811

61. Hsu C W, Septiadi D, Lai C H, Chen P K, Seeberger P H, De Cola L. Glucose-modified silicon nanoparticles for cellular imaging. ChemPlusChem, 2017, 82(4): 660-667

62. Cheng F F, Liang G X, Shen Y Y, Rana R K, Zhu J J. $N$ Acetylglucosamine biofunctionalized $\mathrm{CdSeTe}$ quantum dots as fluorescence probe for specific protein recognition. Analyst (London), 2013, 138(2): 666-670

63. Ahire J H, Chambrier I, Mueller A, Bao Y, Chao Y. Synthesis of Dmannose capped silicon nanoparticles and their interactions with MCF-7 human breast cancerous cells. ACS Applied Materials \& Interfaces, 2013, 5(15): 7384-7391

64. Ahire J H, Behray M, Webster C A, Wang Q, Sherwood V, Saengkrit N, Ruktanonchai U, Woramongkolchai N, Chao Y. Synthesis of carbohydrate capped silicon nanoparticles and their reduced cytotoxicity, in vivo toxicity, and cellular uptake. Advanced Healthcare Materials, 2015, 4(12): 1877-1886

65. Dalal C, Jana N R. Galactose multivalency effect on the cell uptake mechanism of bioconjugated nanoparticles. Journal of Physical Chemistry C, 2018, 122(44): 25651-25660

66. Zayed D G, Ebrahim S M, Helmy M W, Khattab S N, Bahey-El-Din M, Fang J Y, Elkhodairy K A, Elzoghby A O. Combining hydrophilic chemotherapy and hydrophobic phytotherapy via tumor-targeted albumin-QDs nano-hybrids: Covalent coupling and phospholipid complexation approaches. Journal of Nanobiotechnology, 2019, 17(1): 19

67. Yin C, Ying L, Zhang P C, Zhuo R X, Kang E T, Leong K W, Mao $\mathrm{H}$ Q. High density of immobilized galactose ligand enhances hepatocyte attachment and function. Journal of Biomedical Materials Research. Part A, 2003, 67A(4): 1093-1104

68. Hata S, Ishii K. Effect of galactose on binding and endocytosis of asiaioglycoprotein in cultured rat hepatocytes. Annals of Nuclear Medicine, 1998, 12(5): 255-259

69. Mishra N, Yadav N P, Rai V K, Sinha P, Yadav K S, Jain S, Arora S. Efficient hepatic delivery of drugs: Novel strategies and their significance. BioMed Research International, 2013, 2013: 20 
70. Yousef S, Alsaab H O, Sau S, Iyer A K. Development of asialoglycoprotein receptor directed nanoparticles for selective delivery of curcumin derivative to hepatocellular carcinoma. Heliyon, 2018, 4(12): e01071

71. Pranatharthiharan S, Patel M D, Malshe V C, Pujari V, Gorakshakar A, Madkaikar M, Ghosh K, Devarajan P V. Asialoglycoprotein receptor targeted delivery of doxorubicin nanoparticles for hepatocellular carcinoma. Drug Delivery, 2017, 24(1): 20-29

72. Abe M, Manola J B, Oh W K, Parslow D L, George D J, Austin C L, Kantoff P W. Plasma levels of heat shock protein 70 in patients with prostate cancer: A potential biomarker for prostate cancer. Clinical Prostate Cancer, 2004, 3(1): 49-53

73. Ciocca D R, Calderwood S K. Heat shock proteins in cancer: Diagnostic, prognostic, predictive, and treatment implications. Cell Stress \& Chaperones, 2005, 10(2): 86-103

74. Garrido C, Brunet M, Didelot C, Zermati Y, Schmitt E, Kroemer G. Heat shock proteins 27 and 70: Anti-apoptotic proteins with tumorigenic properties. Cell Cycle (Georgetown, Tex.), 2006, 5(22): 2592-2601

75. Ahire J H, Wang Q, Coxon P R, Malhotra G, Brydson R, Chen R, Chao Y. Highly luminescent and nontoxic amine-capped nanoparticles from porous silicon: Synthesis and their use in biomedical imaging. ACS Applied Materials \& Interfaces, 2012, 4(6): 32853292

76. Zhang L W, Monteiro-Riviere N A. Mechanisms of quantum dot nanoparticle cellular uptake. Toxicological Sciences, 2009, 110(1): 138-155

77. Yuan F L, Li S H, Fan Z T, Meng X Y, Fan L Z, Yang S H. Shining carbon dots: Synthesis and biomedical and optoelectronic applications. Nano Today, 2016, 11(5): 565-586

78. Zhang M, Bai L L, Shang W H, Xie W J, Ma H, Fu Y Y, Fang D C, Sun H, Fan L Z, Han M, et al. Facile synthesis of water-soluble, highly fluorescent graphene quantum dots as a robust biological label for stem cells. Journal of Materials Chemistry, 2012, 22(15): 7461-7467

79. Fan Z T, Zhou S X, Garcia C, Fan L Z, Zhou J B. pH-responsive fluorescent graphene quantum dots for fluorescence-guided cancer surgery and diagnosis. Nanoscale, 2017, 9(15): 4928-4933

80. Wang Q, Bao Y, Zhang X, Coxon P R, Jayasooriya U A, Chao Y. Uptake and toxicity studies of poly-acrylic acid functionalized silicon nanoparticles in cultured mammalian cells. Advanced Healthcare Materials, 2012, 1(2): 189-198

81. Park J H, Gu L, von Maltzahn G, Ruoslahti E, Bhatia S N, Sailor M J. Biodegradable luminescent porous silicon nanoparticles for in vivo applications. Nature Materials, 2009, 8(4): 331-336

82. Chen H, Cui S, Tu Z, Gu Y, Chi X. In vivo monitoring of organselective distribution of cdhgte/ $\mathrm{SiO}_{2}$ nanoparticles in mouse model. Journal of Fluorescence, 2012, 22(2): 699-706

83. Qu Y, Li W, Zhou Y, Liu X, Zhang L, Wang L, Li Y F, Iida A, Tang $\mathrm{Z}$, Zhao Y, et al. Full assessment of fate and physiological behavior of quantum dots utilizing caenorhabditis elegans as a model organism. Nano Letters, 2011, 11(8): 3174-3183

84. Schipper M L, Iyer G, Koh A L, Cheng Z, Ebenstein Y, Aharoni A, Keren S, Bentolila L A, Li J, Rao J, et al. Particle size, surface coating, and pegylation influence the biodistribution of quantum dots in living mice. Small, 2009, 5(1): 126-134

85. Choi HS, Liu W, Misra P, Tanaka E, Zimmer J P, Ipe B I, Bawendi M G, Frangioni J V. Renal clearance of quantum dots. Nature Biotechnology, 2007, 25(10): 1165-1170

86. Zhu Y, Hong H, Xu Z P, Li Z, Cai W. Quantum dot-based nanoprobes for in vivo targeted imaging. Current Molecular Medicine, 2013, 13(10): 1549-1567

87. Vela-Ramirez J E, Goodman J T, Boggiatto P M, Roychoudhury R, Pohl N L B, Hostetter J M, Wannemuehler M J, Narasimhan B. Safety and biocompatibility of carbohydrate-functionalized polyanhydride nanoparticles. AAPS Journal, 2015, 17(1): 256-267 